\title{
Origen metabólico y propiedades bioactivas de ácidos grasos ramificados e impares en leche de rumiantes. Revisión
}

Pilar Gómez-Cortés a*

Miguel Ángel de la Fuente ${ }^{a}$

a Instituto de Investigación en Ciencias de la Alimentación (CSIC-UAM), Nicolás Cabrera, 9. Universidad Autónoma de Madrid, 28049 Madrid, España.

* Autor de correspondencia: p.g.cortes@csic.es

\section{Resumen:}

Los ácidos grasos de cadena impar y ramificada (AGCIR) son un grupo de lípidos en la leche que no supera el $5 \%$ de los ácidos grasos (AG) totales, y que agrupa a un conjunto de moléculas entre las cuales los isómeros de los AG pentadecanoico (15:0, iso 15:0 y anteiso 15:0), hexadecanoico (iso 16:0) y heptadecanoico (17:0, iso 17:0 y anteiso 17:0) son los más abundantes. Los AGCIR son sintetizados por microorganismos ruminales a partir de moléculas producidas durante los procesos de la fermentación de alimentos. Investigaciones recientes señalan la posibilidad de síntesis endógena de algunos AG de cadena impar (15:0 y 17:0) y ramificada (iso 17:0 y anteiso 17:0). La presencia de estos $\mathrm{AG}$ en la leche está influenciada por factores dietéticos, principalmente con la proporción de almidón vs fibra, la relación forraje/concentrado y la suplementación con fuentes de grasa que generan cambios en el metabolismo lipídico, que inducen modificaciones en el perfil de AGCIR de la leche. La leche y los productos lácteos son la principal y casi única fuente de AGCIR de la dieta humana. A pesar de su baja concentración, los AGCIR representan propiedades bioactivas que han sido puestas de manifiesto en distintas investigaciones. Este trabajo revisa el origen metabólico, las propiedades bioactivas, así como las más recientes estrategias alimenticias dirigidas para manipular los contenidos y perfiles de AGCIR en la grasa láctea.

Palabras clave: Rumiante, Ácidos grasos, Leche, Productos lácteos, Rumen, Lípidos. 
Recibido: 30/11/2018

Aceptado: 23/10/2019

\section{Introducción}

Los lípidos de la leche se encuentran físicamente en forma de glóbulos formando una emulsión con la fase acuosa. En el interior de estos glóbulos se encuentran los triacilglicéridos (TG), que consisten en moléculas de glicerol esterificadas cada una con tres ácidos grasos (AG). Los TG, al ser los más abundantes (más del $95 \%$ de los lípidos totales), son los principales responsables de las propiedades de los lípidos de la leche, y sus características varían en función de la composición de AG. Aunque la grasa de leche contiene más de 400 AG diferentes ${ }^{(1)}$, apenas 30 o 40 están presentes en concentraciones superiores al $0.1 \%$. El perfil de AG de la leche y los productos lácteos siendo el principal relacionado con factores dietéticos, seguido por la especie de rumiante, y en menor grado, factores genéticos, rendimiento lechero y el estado de lactación.

Considerando su estructura, los AG se dividen en saturados o insaturados. La mayor parte de los AG saturados contienen un número par de átomos de $\mathrm{C}$, con longitudes que van de 4 hasta $20 \mathrm{C}$. Aunque los más abundantes son los que tienen una longitud de cadena de 10 a 20 átomos de $\mathrm{C}$, la grasa de leche de rumiante se caracteriza por la presencia de cantidades importantes de AG de cadena corta, especialmente el 4:0 y el ácido caproico (6:0). Dentro del grupo de insaturados, los cuales pueden tener de uno a cuatro enlaces, el más abundante (15 a $20 \%$ ) es el ácido oleico (cis-9 18:1). La presencia de pequeñas cantidades de ácidos linoleico ( $2 \%$ ) y $\alpha$-linolénico $(0.5 \%)$ en la leche es originaria de la dieta ya que ambos no son sintetizados en los tejidos, por lo que se les da un carácter de AG esenciales.

La leche de rumiantes contiene además AG de número impar de átomos de carbono y los de cadena metil-ramificada (AGCIR). Los de número impar de átomos de $\mathrm{C}$ representan $2 \%$ del total de AG, siendo 15:0 y 17:0 los más abundantes y representativos (Cuadro 1). Los de cadena ramificada se encuentran en una proporción similar y engloban a un mayor número de moléculas, clasificadas como iso y anteiso, con concentraciones variables en productos lácteos. Aunque en conjunto los niveles en grasa láctea de AGCIR no suelen superar el $5 \%$, su presencia tiene gran interés porque se utilizan como indicadores de funcionamiento ruminal $\mathrm{y}$, en humanos, como indicadores de la ingesta de productos lácteos. En comparación con sus homólogos no ramificados, los AG de cadena ramificada presentan puntos de fusión más bajos, especialmente los anteisos, característica que los permite contribuir a mantener la fluidez de la grasa láctea. 
Cuadro 1: Contenido (g/100 de ácidos grasos totales) de ácidos grasos de cadena impar y ramificada en productos lácteos

\begin{tabular}{|c|c|c|c|c|c|c|c|}
\hline \multirow{2}{*}{$\begin{array}{l}\text { Ácido } \\
\text { graso }\end{array}$} & \multicolumn{3}{|c|}{ Leche } & Mant. & Yogurt & Nata & Queso \\
\hline & (7) & (58) & (59) & \multicolumn{4}{|c|}{ (43) } \\
\hline iso & & & 0.04 & & & & \\
\hline 13:0 & & & & & & & \\
\hline $\begin{array}{c}\text { iso } \\
14: 0\end{array}$ & $0.05-0.13$ & & 0.09 & 0.17 & $0.12-0.13$ & $0.00-0.05$ & $0.00-0.22$ \\
\hline $\begin{array}{l}\text { iso } \\
15: 0\end{array}$ & $0.14-0.22$ & & 0.22 & 0.10 & $0.14-0.15$ & $0.00-0.11$ & $0.02-0.42$ \\
\hline $\begin{array}{c}\text { iso } \\
16: 0\end{array}$ & & & 0.21 & 0.34 & $0.29-0.30$ & 0.24 & $0.00-1.18$ \\
\hline $\begin{array}{c}\text { iso } \\
17: 0\end{array}$ & & & 0.27 & 0.31 & $0.16-0.25$ & $0.27-0.30$ & $0.05-0.30$ \\
\hline $\begin{array}{c}\text { iso } \\
18: 0\end{array}$ & & & & $<0.01$ & $0.00-0.04$ & $<0.01$ & $0.00-0.09$ \\
\hline $\begin{array}{c}\text { anteiso } \\
13: 0\end{array}$ & & & 0.08 & & & & \\
\hline $\begin{array}{c}\text { anteiso } \\
15: 0\end{array}$ & $0.32-0.45$ & & 0.46 & 0.63 & $0.62-0.63$ & $0.46-0.49$ & $0.38-0.88$ \\
\hline $\begin{array}{c}\text { anteiso } \\
17: 0\end{array}$ & & & 0.50 & 0.38 & $0.56-0.59$ & $0.36-0.37$ & $0.29-0.61$ \\
\hline $15: 0$ & $0.84-1.31$ & 0.89 & & & & & \\
\hline $17: 0$ & $0.45-0.66$ & 0.52 & $0.55-0.90$ & & & & \\
\hline
\end{tabular}

Aunque una gran proporción de los AGCIR contenidos en la grasa láctea son formados durante los procesos fermentativos en rumen, se ha sugerido recientemente que una pequeña cantidad podría sintetizarse en forma endógena (vgr. tejido mamario). Además, en la última década se han multiplicado las evidencias que apuntan que los AGCIR podrían jugar un papel relevante en la salud humana. Por ello, su presencia en los productos lácteos debería ser contemplada de forma positiva, ya que estos alimentos son casi la única fuente de estos componentes en la dieta. El objetivo de este manuscrito es, por un lado, actualizar la información sobre el origen y la síntesis de estos AG en rumiantes, revisando la influencia del tipo de alimentación sobre su contenido en leche y, por otro, recopilar las evidencias sobre los beneficios nutricionales que la presencia de estos componentes podría reportar en humanos.

\section{Origen de los ácidos grasos de cadena impar y ramificada}

\section{Síntesis ruminal de AGCIR}

Una de las características de la grasa de leche de rumiantes respecto a la de otros mamíferos es la alta concentración de AGCIR. Vlaeminck et al ${ }^{(2)}$ recopilaron datos de 
numerosos estudios sobre la composición de AGCIR en leche y mostraron que los principales AGCIR son isómeros de los AG tetradecanoico (iso 14:0), pentadecanoico (15:0, iso 15:0 y anteiso 15:0), hexadecanoico (iso 16:0) y heptadecanoico (17:0, iso 17:0 y anteiso 17:0).

Los AGCIR son sintetizados principalmente durante los procesos de fermentación microbiana a nivel ruminal. Las bacterias ruminales contienen entre 50 y $90 \mathrm{~g} / \mathrm{kg}$ de lípidos en su materia seca, y alrededor de un $5 \%$ de los mismos son AGCIR que se localizan preferentemente en las membranas ${ }^{(3)}$. Los protozoos poseen menos AGCIR totales que las bacterias (110 vs $160 \mathrm{~g} / \mathrm{kg}$ de AG totales), aunque presentan una mayor proporción de iso 16:0 y anteiso 17:0 $0^{(4)}$.

Los precursores de la síntesis microbiana de AG ramificados en el rumen son leucina, isoleucina, y valina, aminoácidos de cadena ramificada procedentes de la dieta (Figura 1). Inicialmente la microbiota ruminal transforma estos aminoácidos en ácidos carboxílicos ramificados de cadena corta, isovalérico, 2-metil-butírico e isobutírico, respectivamente, unidos a la coenzima A. A continuación, se produce la elongación de cadena de estos AG gracias a la actividad de la enzima AG sintasa (AGS) de los microorganismos. Los iso de cadena par se originan a partir del ácido isobutírico, mientras que los iso y anteiso de cadena impar se generan a partir de los ácidos isovalérico y 2metil-butírico, respectivamente. El precursor de los AG impares de cadena media (13:0, 15:0 y 17:0) en el rumen es el ácido propiónico, que procede de la fermentación de ciertos componentes de la ración, aunque los AG 15:0 y 17:0 también pueden originarse por $\alpha$ oxidación a partir de los AG 16:0 y 18:0 presentes en los lípidos de la dieta.

Figura 1: Síntesis de ácidos grasos de número de carbono impar y de cadena ramificada por la microbiota del rumen

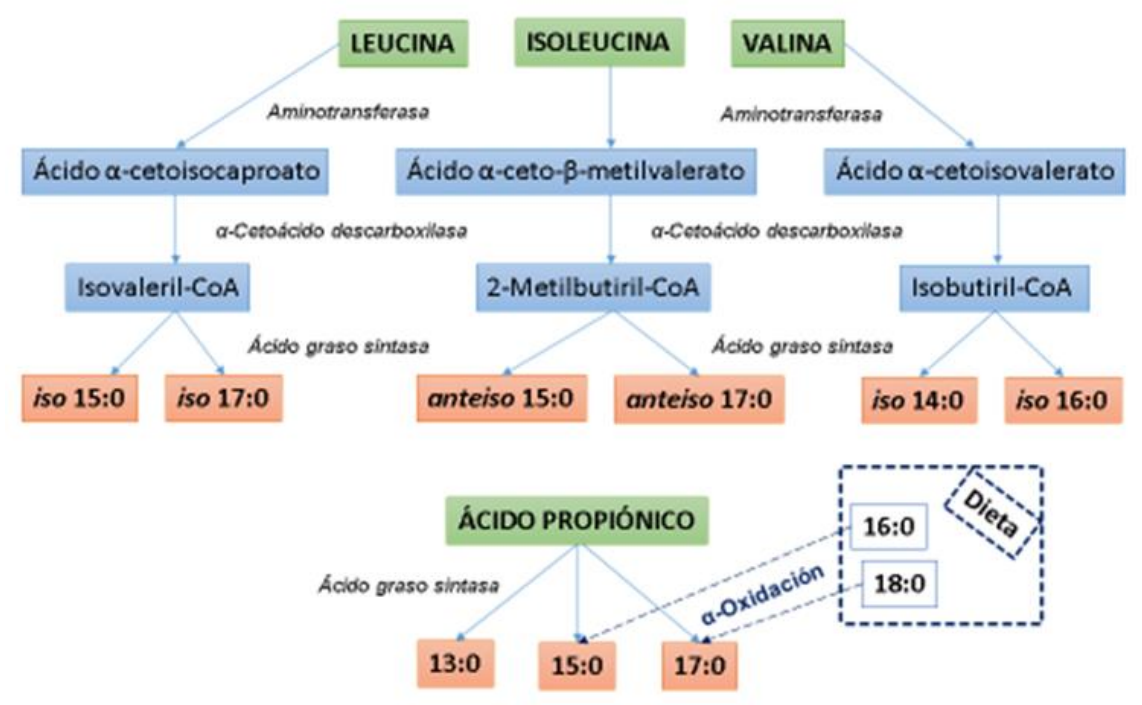

Adaptada de Vlaeminck et al. ${ }^{(2)}$ 
El perfil de AGCIR que se origina tras la digestión ruminal está fuertemente ligado a la actividad de los microorganismos presentes en esta cavidad digestiva ${ }^{(2)}$. De ahí, que la variación en el perfil de AGCIR sea un reflejo de la abundancia relativa de las distintas especies de microorganismos que conforman el ecosistema ruminal ${ }^{(5-6)}$. Las bacterias celulolíticas, es decir, aquellas que poseen enzimas capaces de hidrolizar la celulosa como Ruminococcus flavefaciens, Ruminococcus albus o Butyrivibrio fibrisolvens poseen contenidos destacados de AGCIR $i s o^{(7)}$. Mayores proporciones de anteiso 15:0 indicarían la presencia de bacterias especializadas en la fermentación de pectina y azúcares ${ }^{(8)}$ como $^{(2)}$ Prevotella spp., Lachnospira multiparus y Succinovibrio dextrinosolvens. Las bacterias amilolíticas o digestoras de almidón como Succinivibrio dextrinosolvens, Succinimonas amylolytica, Ruminobacter amylophilus, Selenomonas ruminantium y Streptococcus bovis contienen una menor proporción de AG de cadena ramificada pero destacan por poseer más de cadena impar ${ }^{(2)}$.

\section{Transferencia de AGCIR desde el tracto digestivo a la glándula mamaría}

Es bien conocido el papel preponderante de los microorganismos ruminales en la presencia de AGCIR en productos lácteos ${ }^{(9)}$. Aunque informes recientes ${ }^{(2,10)}$ han cuestionado que la correlación entre el contenido de AGCIR en el fluido intestinal y la grasa láctea sea tan estricta. Teóricamente, se podrían producir desajustes durante la transferencia de estos AG desde el aparato digestivo a los tejidos internos, particularmente en la glándula mamaria. Tales desajustes podrían tener lugar durante el proceso de absorción intestinal o durante el transporte a través del torrente sanguíneo. Al igual que otros AG que alcanzan el intestino delgado, los AGCIR son absorbidos en el yeyuno. Aparentemente la absorción intestinal de los AG de origen microbiano sería más alta ${ }^{(11)}$, pero los pocos datos de los que se dispone no son suficientes para emitir una conclusión definitiva.

Tras su absorción, tanto los AGCIR como el resto de AG, son esterificados en el glicerol para formar TG y fosfolípidos (FL) por las células epiteliales intestinales y transportados, primero al sistema linfático y posteriormente al torrente sanguíneo, donde entran a formar parte de complejos macromoleculares como quilomicrones y lipoproteínas de muy baja densidad (VLDL). Tanto quilomicrones como VLDL contienen distintas clases de lípidos (TG, FL, ésteres de colesterol (EC) y ácidos grasos libres) pero cada una de ellas difiere en composición puesto que cada tipo de AG se enlaza selectivamente a las distintas fracciones. El acceso de los AG desde el torrente sanguíneo al citoplasma de las células de la glándula mamaria se realiza una vez liberados de dichas macromoléculas, a través de la acción de la enzima lipoproteína lipasa (Figura 2). 
Figura 2: Vías metabólicas de síntesis de ácidos grasos en las células de la glándula mamaría de rumiantes

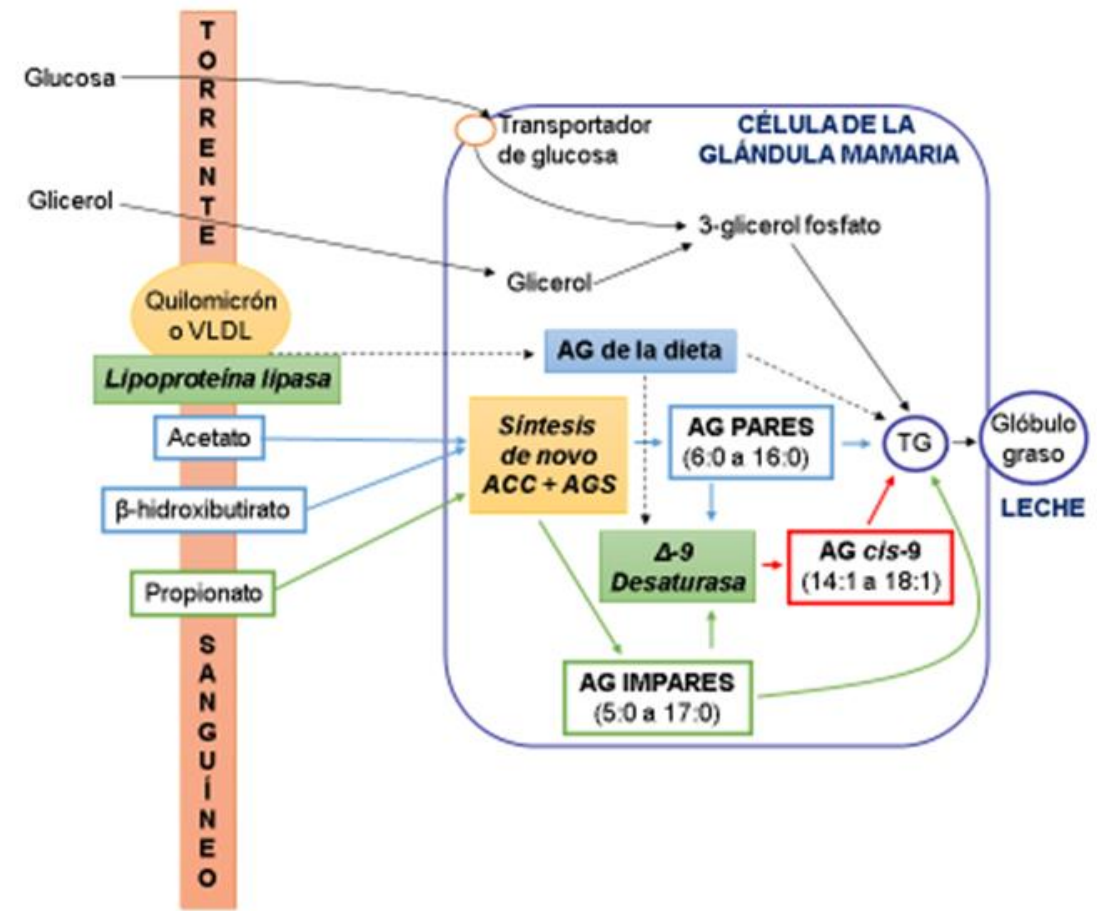

ACC: acetil-CoA carboxilasa; AGS: ácido graso sintasa; AG: ácido graso; TG: triacilglicérido; VLDL: lipoproteínas de muy baja densidad.

La principal diana de la lipoproteína lipasa son los AG de los TG. Por el contrario, los AG característicos de las fracciones de EC y FL se transfieren más pobremente a la grasa de leche debido a la baja afinidad de dicha enzima por ellas. Fievez et al ${ }^{(7)}$ reportaron que los AG de cadena ramificada son más abundantes en los EC y los TG que en los FL o los ácidos grasos libres. Estas dos últimas fracciones, en contraste, son más ricas en AG de cadena impar. Sin embargo, la literatura disponible sobre la distribución de los AGCIR entre los diferentes tipos de lípidos del plasma es aún muy escasa para predecir tendencias o pronosticar comportamientos metabólicos consolidados. Por tanto, merecería la pena explorar en detalle los procesos metabólicos que tienen lugar en las células de la glándula mamaría, para encontrar los mecanismos responsables de las diferencias de los perfiles de AGCIR entre el líquido ruminal y la leche.

\section{Síntesis endógena de AGCIR}

La mayor parte de los AG saturados de número par de átomos de $\mathrm{C}$ presentes en la grasa láctea se sintetizan de novo en las células epiteliales de la glándula mamaria ${ }^{(12)}$. Su síntesis se produce a partir de las moléculas de acetato y $\beta$-hidroxibutirato circulantes en el torrente sanguíneo, generadas en el rumen durante la fermentación de los hidratos de carbono de la dieta. Las dos enzimas responsables de esta síntesis de novo en la glándula mamaria son la acetil-CoA carboxilasa (ACC) y la AGS (Figura 2). El primer paso de la síntesis consiste en la activación del acetato a acetil-CoA, seguido de la condensación de dos moléculas de acetil-CoA para formar malonil-CoA. Esta etapa es catalizada por la 
ACC. A continuación, la AGS regula la elongación de cadena de los AG sintetizados de novo. Si el sustrato inicial en lugar del acetato fuese el propionato, su derivado el metilmalonato, o AG volátiles ramificados (isovalérico, isobutírico y 2-metilbutirico) los productos finales de la síntesis de novo serían potencialmente AG impares, AG metil sustituidos no terminales, o iso y anteiso, respectivamente tal como ocurre en el rumen (Figura 1).

Los primeros trabajos llevados a cabo en este campo demostraron que 15:0 y 17:0 podrían ser sintetizados de novo en la glándula mamaría de rumiantes utilizando propionil-CoA en lugar de acetil-CoA como molécula cebadora o iniciadora ${ }^{(13)}$. La elongación de dicha molécula catalizada por la AGS explicaría la presencia en leche de 5:0, 7:0, 9:0 y 11:0 así como el incremento de las cantidades de 13:0, 15:0 y 17:0 respecto a las ya generadas en el rumen y transferidas desde el duodeno. La importancia de esta síntesis endógena se vio confirmada en trabajos posteriores ${ }^{(10,14,15)}$. Teóricamente estos AG de cadena impar (13:0, 15:0 y 17:0) podrían a su vez ser metabolizados a cis-monoinsaturados por acción de la enzima delta-9 desaturasa. Sin embargo, únicamente la conversión de 17:0 a cis-9 17:1 parece tener importancia cuantitativa ${ }^{(16)}$ (Figura 2).

En contraste a los AG de cadena impar, la síntesis mamaria de los AG iso y anteiso no respondió al aumento de disponibilidad de sus precursores biológicos, los AG isovalérico, 2-metil-butírico e isobutírico ${ }^{(13,14)}$. Esta observación sería un indicio de que la enzima AGS podría no ser activa en el proceso de elongación y, por tanto, la síntesis de novo no tendría lugar en tejidos extraruminales. Estos resultados estarían, sin embargo, en aparente contradicción con los incrementos en los contenidos de iso 17:0 e anteiso 17:0 en grasa láctea, respecto a su presencia en el fluido intestinal, reportados por otros investigadores $^{(2,10,17)}$.

Fievez et $a l^{(7)}$ postularon que los valores más bajos que se desprendían de las relaciones iso 15:0/iso 17:0 y anteiso 15:0/anteiso 17:0 en leche comparadas con las de fluido duodenal podrían ser explicados si se demostrase viable la elongación de cadena de las moléculas de iso 15:0 y anteiso 15:0 una vez absorbidos al torrente sanguíneo. Parecía llamativo en este sentido que la secreción en la leche de iso 15:0 + iso 17:0 y anteiso 15:0 + anteiso 17:0 era muy similar a la suma de estos AG en el duodeno ${ }^{(7)}$. Tales datos reforzaron la hipótesis sobre la existencia de una actividad elongasa extraruminal sobre los AG iso y anteiso de 15 átomos de $\mathrm{C}$ y además apoyó la idea de una transferencia casi completa de los AG de cadena ramificada totales desde el duodeno a la leche. En un estudio posterior Vlaeminck et $a^{(15)}$ observaron mayores niveles de iso 17:0 y anteiso 17:0 en grasa de leche, en comparación con sus contenidos en fluido duodenal (Cuadro 2). Este hecho, junto con unas relaciones iso-15:0/iso-17:0 y anteiso 15:0/anteiso 17:0 más bajas en leche descartarían la síntesis de novo postruminal de estos AG y confirmarían el papel predominante de elongasas post-absorción que ejercerían su actividad sobre los AG iso 15:0 y anteiso 15:0. El valor más bajo de la relación iso 15:0/iso 17:0 y anteiso 15:0/anteiso 17:0 en los TG del plasma sanguíneo, respecto a las 
muestras del fluido duodenal sería además un indicio de que la elongación podría tener lugar en tejidos distintos a la glándula mamaria.

Cuadro 2. Proporción de ácidos grasos del plasma sanguíneo de vacas

\begin{tabular}{|c|c|c|c|c|c|c|}
\hline \multirow{3}{*}{$\begin{array}{l}\text { Acido } \\
\text { graso }\end{array}$} & \multirow[b]{3}{*}{ Ensayo } & \multirow[b]{2}{*}{ Duodeno } & \multirow[b]{2}{*}{ Leche } & \multicolumn{2}{|c|}{ Plasma sanguíneo } & \multirow{3}{*}{$\begin{array}{l}P \text { - } \\
\text { value }\end{array}$} \\
\hline & & & & TG & AGL & \\
\hline & & \multicolumn{4}{|c|}{$\begin{array}{l}\text { g/100 g de ácidos grasos impares y de cadena } \\
\text { ramificada totales }\end{array}$} & \\
\hline \multirow[t]{2}{*}{ iso $15: 0$} & 1 & $12.87^{\mathrm{d}}$ & $7.20^{\mathrm{b}}$ & $9.13^{c}$ & $6.22^{\mathrm{a}}$ & $<0.001$ \\
\hline & 2 & $10.45^{\mathrm{c}}$ & $5.42^{\mathrm{a}}$ & $6.80^{\mathrm{b}}$ & $7.54^{\mathrm{b}}$ & $<0.001$ \\
\hline \multirow{2}{*}{ anteiso 15:0 } & 1 & $26.98^{\mathrm{d}}$ & $14.54^{\mathrm{b}}$ & $19.07^{\mathrm{c}}$ & $12.47^{\mathrm{a}}$ & $<0.001$ \\
\hline & 2 & $33.57^{\mathrm{c}}$ & $13.22^{\mathrm{a}}$ & $15.00^{\mathrm{ab}}$ & $19.06^{\mathrm{b}}$ & $<0.001$ \\
\hline \multirow[t]{2}{*}{ iso $17: 0$} & 1 & $5.76^{\mathrm{a}}$ & $8.85^{\mathrm{b}}$ & $10.02^{c}$ & $13.45^{\mathrm{d}}$ & $<0.001$ \\
\hline & 2 & $5.79^{\mathrm{a}}$ & $6.64^{\mathrm{a}}$ & $9.54^{\mathrm{b}}$ & $10.09^{b}$ & $<0.001$ \\
\hline \multirow[t]{2}{*}{ anteiso 17:0 } & 1 & $7.32^{\mathrm{a}}$ & $13.24^{\mathrm{b}}$ & $14.21^{\mathrm{bc}}$ & $15.50^{\mathrm{c}}$ & $<0.001$ \\
\hline & 2 & $9.90^{\mathrm{a}}$ & $16.18^{\mathrm{bc}}$ & $14.41^{\mathrm{b}}$ & $17.63^{\mathrm{c}}$ & $<0.001$ \\
\hline \multicolumn{7}{|c|}{ Relaciones C15/C17 } \\
\hline \multirow{3}{*}{$\begin{array}{ll}\text { iso } & 1 \\
17: 0\end{array}$} & 1 & $2.28^{c}$ & $0.83^{\mathrm{b}}$ & $0.92^{b}$ & $0.48^{\mathrm{a}}$ & $<0.001$ \\
\hline & & & & & & \\
\hline & 2 & $1.82^{b}$ & $1.18^{\mathrm{ab}}$ & $0.78^{\mathrm{a}}$ & $0.77^{\mathrm{a}}$ & $<0.001$ \\
\hline \multirow{2}{*}{$\begin{array}{l}\text { ant } \\
17: 0\end{array}$} & 1 & $3.98^{b}$ & $1.10^{\mathrm{a}}$ & $1.37^{\mathrm{a}}$ & $0.82^{\mathrm{a}}$ & $<0.001$ \\
\hline & 2 & $3.73^{\mathrm{a}}$ & $0.84^{\mathrm{b}}$ & $1.10^{\mathrm{b}}$ & $1.13^{\mathrm{b}}$ & $<0.001$ \\
\hline
\end{tabular}

La sobreexpresión del gen que codifica la elongasa ELOVL6 en rumiantes está descrita en células epiteliales mamarias ${ }^{(18)}$, y, más recientemente, un estudio in vitro ha sido el primero en valorar el papel de esta enzima en la regulación de la elongación de $\mathrm{AG}^{(19)}$. La sobrerregulación de ELOVL6 aumenta los índices de elongación de 16:0 y 18:0, lo que sugiere un papel importante de este enzima en el control de longitud de cadena de los AG en la glándula mamaría. Sin embargo, sus efectos sobre los AG ramificados todavía quedan pendientes por ser investigados.

\section{Influencia de la dieta del ganado sobre los contenidos de AGCIR en leche}

La composición química de la ración, tal como la proporción de almidón y fibra, la relación forraje/concentrado (F/C), así como el perfil lipídico en la dieta ejercen una gran influencia sobre el tipo de poblaciones microbianas ruminales y sobre la síntesis microbiana de AG, por lo tanto, la proporción de AGCIR que llega a intestino delgado es un reflejo de la composición y cantidad de microbiota en rumen ${ }^{(2,3,20)}$. 


\section{Efectos de la dieta base}

Entre los distintos componentes de la dieta, la proporción de almidón y fibra juega un papel relevante en la producción de AGCIR a través de su influencia en el ecosistema microbiano, en particular sobre la proliferación de cepas bacterianas celulolíticas ${ }^{(21,22)}$. Un aumento de almidón en las raciones limita el crecimiento de microorganismos celulolíticos, promoviendo la proliferación de bacterias amilolíticas. Como ya se expuso, las bacterias celulolíticas contienen en sus membranas predominantemente AG ramificados $i s o^{(7)}$ y son sensibles a valores bajos de $\mathrm{pH}$ ruminal que son los característicos de alimentos con alto contenido de almidón ${ }^{(23)}$. Vlaeminck et al ${ }^{(2)}$ observaron que dietas ricas en almidón, caracterizadas por una mayor proliferación de bacterias amilolíticas, disminuían los niveles de iso 14:0, iso 15:0 e iso 16:0 (Cuadro 3).

Cuadro 3: Contenido medio ( $\mathrm{g} / 100 \mathrm{~g}$ de ácidos grasos totales) de ácidos grasos de cadena impar y ramificada en leche de rumiantes alimentados con diversos ingredientes

\begin{tabular}{|c|c|c|c|c|c|c|c|c|c|c|}
\hline \multirow[t]{2}{*}{$\begin{array}{l}\text { Acido } \\
\text { graso }\end{array}$} & \multicolumn{2}{|c|}{$\begin{array}{l}\text { Shingfield et } \\
a l^{(60)}\end{array}$} & \multicolumn{2}{|c|}{$\begin{array}{l}\text { Vlaeminck et } \\
a l^{(2)}\end{array}$} & \multicolumn{2}{|c|}{ Patel et $a l^{(24)}$} & \multicolumn{2}{|c|}{ Li et $a l^{(25)}$} & \multicolumn{2}{|c|}{$\begin{array}{l}\text { Cívico et } \\
a l^{(27)}\end{array}$} \\
\hline & EP & EM & EP & EM & EPA & EPB & $\mathbf{R F}$ & PF & RF & RA \\
\hline iso $13: 0$ & 0.03 & 0.04 & & & & & & & 0.02 & 0.01 \\
\hline iso $14: 0$ & 0.08 & 0.06 & 0.09 & 0.05 & 0.08 & 0.07 & 0.14 & 0.13 & 0.07 & 0.03 \\
\hline iso $15: 0$ & 0.21 & 0.18 & 0.24 & 0.17 & 0.21 & 0.18 & 0.32 & 0.23 & 0.17 & 0.14 \\
\hline iso $16: 0$ & 0.21 & 0.23 & 0.18 & 0.16 & 0.26 & 0.26 & & & 0.19 & 0.15 \\
\hline iso $17: 0$ & 0.74 & 0.91 & 0.19 & 0.23 & 0.47 & 0.33 & 0.38 & 0.49 & 0.27 & 0.23 \\
\hline iso $18: 0$ & 0.03 & 0.01 & & & & & & & 0.05 & 0.04 \\
\hline anteiso & 0.05 & 0.07 & & & & & & & & \\
\hline $13: 0$ & & & & & & & & & & \\
\hline $\begin{array}{l}\text { anteiso } \\
15: 0\end{array}$ & 0.39 & 0.37 & 0.46 & 0.46 & 0.42 & 0.39 & 0.49 & 0.45 & 0.30 & 0.22 \\
\hline anteiso & & & 0.46 & 0.55 & & & 0.89 & 0.76 & 0.29 & 0.26 \\
\hline $17: 0$ & & & & & & & & & & \\
\hline 11:0 & & & & & & & 0.20 & 0.22 & & \\
\hline $13: 0$ & & & & & & & 0.09 & 0.09 & & \\
\hline $15: 0$ & 1.22 & 0.78 & 0.95 & 1.21 & 1.06 & 0.94 & 1.00 & 0.98 & 0.82 & 0.62 \\
\hline $17: 0$ & 0.63 & 0.54 & 0.48 & 0.55 & 0.67 & 0.53 & 0.73 & 0.68 & & \\
\hline
\end{tabular}

$\mathrm{EP}=$ ensilado de pasto; $\mathrm{EM}=$ ensilado de maíz; $\mathrm{EPA}=$ ensilado de pasto alto; $\mathrm{EPB}=$ ensilado de pasto bajo; $\mathrm{RF}=$ rico en fibra; $\mathrm{PF}=$ pobre en fibra; $\mathrm{RA}=$ rico en almidón.

Estudios posteriores han reforzado la idea de la influencia que la proporción de fibra y almidón tiene sobre el contenido de AGCIR de la leche (Cuadro 3). Patel et al ${ }^{(24)}$ reportaron que un aumento de fibra a través de la presencia de ensilados de pasto en las raciones aumentaba los contenidos en leche de iso 15:0, iso 17:0, 15:0 y 17:0 mientras que la sustitución de fibra en detrimento de almidón en la dieta incrementaba la proporción de iso 15:0 tanto en leche ${ }^{(25)}$ como en el rumen ${ }^{(26)}$. Estas respuestas se 
asociaron a una mayor abundancia de bacterias celulolíticas frente a las amilolíticas. Por otra parte, Cívico et al ${ }^{(27)}$ midieron niveles más elevados de iso 14:0, iso 17:0 y 15:0 en grasa láctea cuando la alimentación estaba enriquecida en fibra y era pobre en almidón (Cuadro 3).

La relación F/C en las raciones podría modificar los contenidos de AGCIR de los productos lácteos. Vlaeminck et $a l^{(2)}$ concluyeron que una proporción mayor de forraje en la dieta base contribuía a un aumento selectivo de ciertos AGCIR como iso 14:0 e iso 15:0. En contraste, los niveles de anteiso 15:0 fueron menos afectados. Estos resultados se explican por cambios en el ecosistema ruminal inducidos por la variación en la relación F/C de las dietas. Un aumento de concentrado favorecería la proliferación de bacterias amilolíticas, lo cual podría aumentar los anteisos y los de cadena impar. Se han observado $^{(10)}$ niveles más bajos de 15:0 y 17:0 en leche de vacas alimentadas con dietas con una elevada relación F/C.

El análisis de fluidos digestivos extraídos de cabras canuladas duodenalmente constató que el aumento de la relación F/C en la ración causaba un aumento de todos los AGCIR sintetizados de novo por las bacterias ${ }^{(5)}$. Un experimento similar llevado a cabo en vacuno $^{(28)}$ mostró resultados parecidos. Más recientemente, Zhang et al ${ }^{(29)}$ confirmaron que los perfiles de AGCIR en fluidos digestivos en bovino son afectados de forma drástica por la relación F/C de la dieta base. Las concentraciones de 11:0, 13:0, iso 15:0, iso 16:0, iso 17:0 y 17:0 eran más altas cuando la proporción de forraje en la ración era mayor. También observaron que sólo los anteiso 15:0 y 15:0 aumentaban con proporciones más elevadas de concentrado.

\section{Efectos de la suplementación lipídica}

Los niveles de AGCIR en productos lácteos muestran una disminución significativa cuando proceden de animales cuya dieta ha sido suplementada con fuentes lipídicas. Esta pauta, observada en leche bovina ${ }^{(6,30)}$ y de pequeños rumiantes ${ }^{(31,32)}$ es característica de la suplementación con semillas de oleaginosas ricas en AG insaturados.

Estos resultados podrían ser explicados por el efecto inhibitorio que los ácidos grasos poliinsaturados (PUFA) ejercen sobre la microbiota intestinal. La severidad del efecto de los AG incorporados de la dieta sobre la viabilidad de las bacterias del rumen es mayor a medida que aumenta el número de insaturaciones de las moléculas. Así mismo los efectos serían más acusados si la configuración geométrica de los dobles enlaces es de tipo cis $^{(2,3)}$. Además, no todos los microorganismos se verían afectados de la misma forma por la suplementación lipídica de la dieta. Se ha observado que las bacterias celulolíticas y Gram positivas son más sensibles a los lípidos dietéticos que las bacterias amilolíticas y Gram negativas $^{(20,33,34)}$. 


\section{Ácidos grasos ramificados como componentes bioactivos}

\section{Microbiota intestinal de neonatos}

Estudios recientes han destacado el papel de los AG ramificados como componentes bioactivos protectores de la salud. La presencia de AG ramificados es muy escasa en los tejidos humanos de adultos pero, en contraste, son componentes bioactivos esenciales del tracto digestivo en los estadios finales del desarrollo fetal y tras el parto ${ }^{(35)}$.

Aproximadamente, $30 \%$ de los AG totales presentes en el vérnix caseoso son AG de cadena ramificada, con una gran variedad de estructuras moleculares entre las que destacan el iso 14:0 y el iso 16:0 $0^{(35)}$. El vérnix es un material ceroso con una textura similar al queso que reviste la piel del feto y del recién nacido. Consiste en una mezcla de secreciones grasas originadas a partir de la 18 semana de gestación procedentes de las glándulas sebáceas. El vérnix evita la pérdida de agua, protegiendo la piel del feto de la deshidratación, impidiendo su endurecimiento y disminuyendo las rozaduras y el agrietamiento. Además, contribuye a regular la temperatura del feto al actuar como una capa aislante. No existe otro mamífero terrestre que produzca neonatos cubiertos de vérnix, en contraste a lo que ocurre con los fetos de mamíferos acuáticos que presentan esta misma película grasa compuesta por AG ramificados ${ }^{(36)}$.

Una hipótesis complementaria señala el papel del vérnix como agente antibacteriano. Esta idea se basa en que, durante los últimos meses del embarazo, las partículas de vérnix se desprenden de la piel y pasan al líquido amniótico incrementando su turbidez. En el último trimestre de gestación el feto ingiere buena parte del líquido amniótico y, de esta forma, su intestino quedaría impregnado de los AG ramificados presentes en el vérnix ${ }^{(35)}$.

Por otra parte, la gran cantidad de AG ramificados detectados en el meconio (las primeras heces fecales del neonato) constituye un indicio suficientemente relevante sobre el tipo de microorganismos que comienzan a colonizar el tracto intestinal del recién nacido y que se vería favorecido por la presencia de estos prebióticos no fermentables ${ }^{(35)}$. Como se ha expuesto en párrafos precedentes, los AG ramificados están entre las moléculas más importantes de las membranas de muchos microorganismos, particularmente de la mayoría de las especies del genero bacilli ${ }^{(37)}$. Se ha observado que la sustitución de grasa de la dieta por AG ramificados en crías de ratas neonatas favorece la alteración de su microbioma. Estos cambios se traducen en aumentos de los microorganismos capaces de incorporar AG ramificados en sus membranas y una reducción simultánea de la incidencia de enterocolitis necrotizante ${ }^{(38)}$, una de las mayores causas de mortalidad en neonatos prematuros. También se sabe por estudios in vitro que los AG ramificados reducirían la motilidad y presumiblemente la virulencia de patógenos como Pseudomonas aeruginosa $^{(39)}$. 
La elevada concentración de AG ramificados, como consecuencia de la presencia del vérnix en el lumen intestinal del feto, es probable que juegue además un papel importante en el crecimiento y metabolismo de los enterocitos, así como en la salud y la regulación intestinal. Trabajos recientes han observado que los AG ramificados pueden ser incorporados a los FL de las membranas de los enterocitos, confiriéndoles actividad antiinflamatoria ${ }^{(40,41)}$. Liu et al $^{(42)}$ postularon que esta incorporación de los AG ramificados en los FL contribuiría a modular las propiedades biofísicas de las membranas. Los AG ramificados tienen asignadas funciones biofísicas equiparables a los AG monoinsaturados con configuración cis pero tienen la ventaja de presentar una mayor estabilidad oxidativa por la ausencia de dobles enlaces en su estructura. Por otra parte, los menores puntos de fusión de los AG ramificados respecto a sus homólogos lineales estarían relacionados con el mantenimiento de la fluidez de las membranas celulares ${ }^{(43)}$.

\section{Otras propiedades bioactivas de los ácidos grasos de cadena ramificada}

Además de sus efectos positivos sobre la composición de la microbiota intestinal, la presencia de AG ramificados en la dieta podría redundar beneficiosamente en la prevención de distintas enfermedades. El primer trabajo donde se atribuye actividad anticancerígena a los AG ramificados data de principios de este siglo ${ }^{(44)}$. En dicho estudio se describen los efectos inhibitorios de iso 15:0 sobre la proliferación celular y la inducción de la apoptosis en líneas celulares de cáncer de próstata, leucemia y adenocarcinoma. Más recientemente, Cai et al ${ }^{(45)}$ dieron cuenta de que el iso 15:0 podría contribuir a la inhibición de linfomas humanos. Estudios desarrollados por otros investigadores ${ }^{(46,47)}$ determinaron que los $\mathrm{AG}$ ramificados podrían también inducir apoptosis en células de cáncer de mama e inhibir el desarrollo de tumores en cultivos celulares y modelos animales.

Por otro lado, un reciente estudio en humanos con sobrepeso ${ }^{(48)}$ ha apuntado por primera vez la posibilidad de que la abundancia de AG ramificados iso en suero sanguíneo pueda correlacionarse inversamente con la presencia de TG y asociarse negativamente con otros indicadores característicos de procesos inflamatorios. En todo caso, los efectos beneficiosos de este grupo de AG precisan más investigaciones que contribuyan a esclarecer los mecanismos que subyacen en la prevención de estas patologías.

\section{Ácidos grasos de cadena impar como componentes bioactivos}

Distintos trabajos desarrollados durante estos últimos años han mostrado que 15:0 y 17:0, los AG impares más abundantes en productos lácteos, podrían ejercer efectos saludables en la salud humana ${ }^{(49,50)}$. Se ha comprobado, por ejemplo, que existe una asociación inversa entre la concentración de ambos AG en plasma y el riesgo de padecer diabetes tipo $2^{(51-53)}$. Este resultado se ha observado también en poblaciones europeas sometidas a distintos regímenes alimentarios ${ }^{(54)}$. Incluso varios estudios de prospectiva sobre 
enfermedades cardiovasculares han comprobado que la concentración en plasma de estos AG estaría asociada con un riesgo más bajo de enfermedades cardiovasculares ${ }^{(55-57)}$. Sin embargo, se precisa una investigación más detallada que contribuya a dilucidar las rutas metabólicas implicadas en estos efectos saludables.

\section{Conclusiones}

La leche y los productos lácteos son la mayor fuente de AGCIR en la dieta humana. A pesar de sus bajas concentraciones, investigaciones llevadas a cabo durante los últimos años han mostrado su potencialidad como componentes bioactivos y su relevancia nutricional. Aunque su origen reside principalmente en la actividad de la microbiota ruminal, los últimos avances científicos no circunscriben su formación únicamente a los procesos bioquímicos que tienen lugar en el tracto digestivo de los rumiantes. La capacidad de otros tejidos de sintetizar endógenamente ciertos AGCIR debe ser considerada con atención y puede incentivar líneas de investigación muy prometedoras en el futuro.

\section{Conflicto de interés}

Los autores declaran no tener conflictos de interés.

\section{Literatura citada:}

1. Schroeder M, Vetter W. Detection of 430 Fatty acid methyl esters from transesterified butter sample. J Am Oil Chem Soc 2013;90:771-790.

2. Vlaeminck B, Fievez V, van Laar H, Vlaeminck B, Fievez V, Cabrita ARJ, Fonseca AJM et al. Factors affecting odd- and branched-chain fatty acids in milk: A review. Anim Feed Sci Technol 2006;131:389-417.

3. Buccioni A, Decandia M, Minieri S, Molle G, Cabiddu, A. Lipid metabolism in the rumen: New insights on lipolysis and biohydrogenation with an emphasis on the role of endogenous plant factors. Anim Feed Sci Technol 2012;174:1-25.

4. Or-Rashid MM, Odongo NE, McBride BW. Fatty acid composition of ruminal bacteria and protozoa, with emphasis on conjugated linoleic acid vaccenic acid, and oddchain and branched-chain fatty acids. J Animal Sci 2007;85:1228-1234.

5. Bas P, Archimède H, Rouzeau A, Sauvant, D. Fatty acid composition of mixed-rumen bacteria: effect of concentration and type of forage. J Dairy Sci 2003;86:2940-2948.

6. Baumann E, Chouinard PY, Lebeuf Y, Rico DE, Gervais R. Effect of lipid supplementation on milk odd- and branched-chain fatty acids in dairy cows. J Dairy Sci 2016;99:6311-6323. 
7. Fievez V, Colman E, Castro-Montoya JM, Stefanov I, Vlaeminck B. Milk odd- and branched-chain fatty acids as biomarkers of rumen function-An update. Anim Feed Sci Technol 2012;172:51-65.

8. Bessa RJB, Maia, MRG, Jerónimo E, Belo AT, Cabrita ARJ, Dewhurst RJ, Fonseca AJM. Using microbial fatty acids to improve understanding of the contribution of solid associated bacteria to microbial mass in the rumen. Anim Feed Sci Technol 2009;150:197-206.

9. Keeney M, Katz I, Allison J. On the probable origin of milk fat acids in rumen microbial lipids. J Am Oil Chem Soc 1962;39:198-201.

10. Dewhurst RJ, Moorby JM, Vlaeminck B, Van Nespen T, Fievez V. Apparent recovery of duodenal odd- and branched-chain fatty acids in milk. J Dairy Sci 2007;90:17751780.

11. Schmidely P, Glasser F, Doreau M, Sauvant D. Digestion of fatty acids in ruminants: a meta-analysis of flows and variation factors. 1. Total fatty acids. Animal 2008;2:677-690.

12. Palmquist DL. Milk fat: Origin of fatty acids and influence of nutritional factors thereon. In: Fox PF, McSweeney PLH editors. Advanced dairy chemistry, vol 2, Lipids, $3^{\text {rd }}$ ed. New York, USA: Springer; 2006:43-92.

13. Massart-Leën AM, Roets E, Peeters G, Verbeke R. Propionate for fatty acid synthesis by the mammary gland of the lactating goat. J Dairy Sci 1983;66:1445-1454.

14. French EA, Bertics SJ, Armentano, LE. Rumen and milk odd-and branched-chain fatty acid proportions are minimally influenced by ruminal volatile fatty acid infusions. J Dairy Sci 2012;95:2015-2026.

15. Vlaeminck B, Gervais R, Rahman MM, Gadeyne F, Gorniak, M, Doreau M et al. Postruminal synthesis modifies the odd- and branched chain fatty acid profile from the duodenum to milk. J Dairy Sci 2015;98:4829-4840.

16. Fievez V, Vlaeminck B, Dhanoa MS, Dewhurst RJ. Use of principal component analysis to investigate the origin of heptadecenoic and conjugated linoleic acids in milk. J Dairy Sci 2003;86:4047-4053.

17. Gervais R, Vlaeminck B, Fanchone A, Nozière P, Doreau M, Fievez V. Odd-and branched-chain fatty acids duodenal flows and milk yields in response to $\mathrm{N}$ underfeeding and energy source in dairy cows. J Dairy Sci 2011;94(Suppl 1):125126.

18. Xu HF, Luo J, Zhao WS, Yang YC, Tian HB, Shi HB et al. Overexpression of SREBP1 (sterol regulatory element binding protein 1) promotes de novo fatty acid synthesis and triacylglycerol accumulation in goat mammary epithelial cells. J Dairy Sci 2016;99:783-795. 
19. Shi HB, Wu M, Zhu JJ, Zhang CH, Yao, DW, Luo J et al. Fatty acid elongase 6 plays a role in the synthesis of long-chain fatty acids in goat mammary epithelial cells. $\mathrm{J}$ Dairy Sci 2017;100:4987-4995.

20. Enjalbert F, Combes S, Zened A, Meynadier A. Rumen microbiota and dietary fat: a mutual shaping. J Appl Microbiol 2017;123:782-797.

21. Vlaeminck B, Dewhurst RJ, Demeyer D, Fievez V. Odd and branched chain fatty acids to estimate proportions of cellulolytic and amylolytic particle associated bacteria. J Anim Feed Sci 2004;13:235-238.

22. Cabrita ARJ, Vale JMP, Bessa RJB, Dewhurst RJ, Fonseca AJM. Effects of dietary starch source and buffers on milk responses and rumen fatty acid biohydrogenation in dairy cows fed maize silage-based diets. Anim Feed Sci Technol 2009;52:267277.

23.Sun YZ, Mao SY, Zhu WY. Rumen chemical and bacterial changes during stepwise adaptation to a high-concentrate diet in goats. Animal 2010;4:210-217.

24. Patel M, Wredle E, Bertilsson J. Effect of dietary proportion of grass silage on milk fat with emphasis on odd- and branched-chain fatty acids in dairy cows. J Dairy Sci 2013;96:390-397.

25. Li F, Li Z, Li S, Ferguson JD, Cao Y, Yao J et al. Effect of dietary physically effective fiber on ruminal fermentation and the fatty acid profile of milk in dairy goats. J Dairy Sci 2014;97:2281-2290.

26. Li F, Yang XJ, Cao YC, Li SX, Yao JH, Li ZJ et al. Effects of dietary effective fiber to rumen degradable starch ratios on the risk of sub-acute ruminal acidosis and rumen content fatty acids composition in dairy goat. Anim Feed Sci Technol 2014;189:5462.

27. Cívico A, Núñez Sánchez N, Gómez-Cortés P, De la Fuente MA, Peña Blanco F, Juárez $\mathrm{M}$ et al. Odd- and branched-chain fatty acids in goat milk as indicators of diet composition. Ital J Anim Sci 2017;16:68-74.

28. Vlaeminck B, Fievez V, Demeyer D, Dewhurst RJ. Effect of forage:concentrate ratio on fatty acid composition of rumen bacteria isolated from ruminal and duodenal digesta. J Dairy Sci 2006;89:2668-2678.

29. Zhang Y, Liu K, Hao X, Xin H. The relationships between odd- and branched-chain fatty acids to ruminal fermentation parameters and bacterial populations with different dietary ratios of forage and concentrate. J Anim Physiol Anim Nutr 2017;101:1103-1114. 
30. Vazirigohar M, Dehghan-Banadaky M, Rezayazdi K, Nejati-Javaremi A, MirzaeiAlamouti H, Patra AK. Effects of diets containing supplemental fats on ruminal fermentation and milk odd- and branched-chain fatty acids in dairy cows. J Dairy Sci 2018;101:6133-6141.

31. Gómez-Cortés P, Toral PG, Frutos P, Juárez M, De la Fuente MA, Hervás, G. Effect of the supplementation of dairy sheep diet with incremental amounts of sunflower oil on animal performance and milk fatty acid profile. Food Chem 2011;125:644651.

32. Martínez-Marín AL, Gómez-Cortés P, Gómez CG, Juárez M, Pérez AL, Pérez HM et al. Animal performance and milk fatty acid profile of dairy goats fed diets added differently unsaturated plant oils on fatty acid profile of goat milk. J Dairy Sci 2011;94:5359-5368.

33. Maia MRG, Chaudhary LC, Figueres L, Wallace RJ. Metabolism of polyunsaturated fatty acids and their toxicity to the microflora of the rumen. Antonie van Leeuwenhoek 2007;91:303-314.

34. Yang SL, Bu DP, Wang JQ, Hu ZY, Li D, Wei HY, et al. Soybean oil and linseed oil supplementation affect profiles of ruminal microorganisms in dairy cows. Animal 2009;3:1562-1569.

35. Ran-Ressler RR, Devapatla S, Lawrence P, Brenna JT. Branched chain fatty acids are constituents of the normal healthy newborn gastrointestinal tract. Pediatr Res 2008;64:605-609.

36. Wang DH, Ran-Ressler R, St Leger J, Nilson E, Palmer L, Collins R et al. Sea lions develop human-like vernix caseosa delivering branched fats and squalene to the GI tract. Sci Rep 2018;8:7478.

37. Kaneda T. Fatty acids of the genus bacillus: An example of branched-chain preference. Bacteriol Rev 1977;41:391.

38. Ran-Ressler RR, Khailova L, Arganbright KM, Adkins-Rieck CK, Jouni ZE, Koren $\mathrm{O}$ et al. Branched chain fatty acids reduce the incidence of necrotizing enterocolitis and alter gastrointestinal microbial ecology in a neonatal rat model. PLoS One 2011;6:e29032.

39. Inoue T, Shingaki R, Fukui K. Inhibition of swarming motility of Pseudomonas aeruginosa by branched-chain fatty acids. FEMS Microbiol Lett 2008;281:81-86.

40. Yan Y, Wang Z, Greenwald J, Kothapalli KSD, Park HG, Liu R et al. BCFA suppresses LPS induced IL-8 mRNA expression in human intestinal epithelial cells. Prostaglandins Leukot Essent Fatty Acids 2017;116:27-31. 
41. Yan Y, Wang Z, Wang D, Lawrence P, Wang X, Kothapalli KSD et al. BCFAenriched vernix-monoacylglycerol reduces LPS-induced inflammatory markers in human enterocytes in vitro. Pediatr Res 2018;83:874-879.

42. Liu L, Wang Z, Park HG, Xu C, Lawrence P, Sub X et al. Human fetal intestinal epithelial cells metabolize and incorporate branched chain fatty acids in a structure specific manner. Prostaglandins Leukotr Essent Fatty Acids 2017;116:32-39.

43. Ran-Ressler RR, Bae S, Lawrence P, Wang DH, Brenna JT. Branched chain fatty acid content of foods and estimated intake in the USA. Br J Nutr 2014;112:565-572.

44. Yang Z, Liu S, Chen X, Chen H, Huang M, Zheng J. Induction of apoptotic cell death and in vivo growth inhibition of human cancer cells by a saturated branched-chain fatty acid, 13-methyltetradecanoic acid. Cancer Res 2000;60:505-509.

45. Cai Q, Huang H, Qian D, Chen K, Luo J, Tian Y et al. 13- methyltetradecanoic acid exhibits antitumor activity on T-cell lymphomas in vitro and in vivo by downregulating p-AKT and activating caspase-3. PLoS One 2013;8:e65308.

46. Wongtangtintharn S, Oku H, Iwasaki H, Toda T. Effect of branched-chain fatty acids on fatty acid biosynthesis of human breast cancer cells. J Nutr Sci Vitaminol 2004;50:137-143.

47. Wongtangtintharn $\mathrm{S}$, Oku $\mathrm{H}$, Iwasaki $\mathrm{H}$, Inafuku $\mathrm{M}$, Toda $\mathrm{T}$, Yanagita $\mathrm{T}$. Incorporation of branched-chain fatty acid into cellular lipids and caspase in dependent apoptosis in human breast cancer cell line, SKBR-3. Lipids Health Dis 2005;4:29.

48. Mika A, Stepnowski P, Kaska L, Proczko M, Wisniewski P, Sledzinski M et al. A Comprehensive study of serum odd- and branched-chain fatty acids in patients with excess weight. Obesity 2016;24:1669-1676.

49. Jenkins B, West JA, Koulman A. A review of odd-chain fatty acid metabolism and the role of pentadecanoic acid (C15:0) and heptadecanoic acid (C17:0) in health and disease. Molecules 2015;20:2425-2444.

50. Jenkins BJ, Seyssel K, Chiu S, Pan PH, Lin SY, Stanley E et al. Odd chain fatty acids; New insights of the relationship between the gut microbiota, dietary intake, biosynthesis and glucose intolerance. Sci Rep 2017;7:44845.

51. Yakoob MY, Shi PL, Willett WC, Rexrode KM, Campos H, Orav EJ et al. Circulating biomarkers of dairy fat and risk of incident diabetes mellitus among men and women in the United States in two large prospective cohorts. Circulation 2016;133:16451654.

52.Pfeuffer M, Jaudszus A. Pentadecanoic and heptadecanoic acids: multifaceted oddchain fatty acids. Adv Nutr 2016;7:730-734. 
53. Risérus U, Marklund M. Milk fat biomarkers and cardiometabolic disease. Curr Opin Lipidol 2017;28:46-51.

54. Forouhi NG, Koulman A, Sharp SJ, Imamura F, Kroger J, Schulze MB et al. Differences in the prospective association between individual plasma phospholipid saturated fatty acids and incident type 2 diabetes: the EPIC-InterAct case-cohort study. Lancet Diabetes Endocrinol 2014;2:810-818.

55. Khaw KT, Friesen MD, Riboli E, Luben R, Wareham N. Plasma phospholipid fatty acid concentration and incident coronary heart disease in men and women: The EPIC-Norfolk prospective study. PLoS Med 2012;9:e1001255.

56. Otto MCD, Nettleton JA, Lemaitre RN, Steffen LM, Kromhout D, Rich SS et al. Biomarkers of dairy fatty acids and risk of cardiovascular disease in the multi-ethnic etudy of atherosclerosis. J Am Heart Assoc 2013;2:e000092.

57. Liang J, Zhou Q, Amakye WK, Su Y, Zhang Z. Biomarkers of dairy fat intake and risk of cardiovascular disease: A systematic review and meta analysis of prospective studies. Crit Rev Food Sci Nutr 2018;58:1122-1130.

58. O'Donnell-Megaro AM, Barbano DM, Bauman DE. Survey of the fatty acid composition of retail milk in the United States including regional and seasonal variations. J Dairy Sci 2011;94:59-65.

59. Shingfield KJ, Chilliard Y, Toivonen P, Kairenius P, Givens DI. Trans fatty acids and bioactive lipids in ruminant milk. Adv Exp Med Biol 2008;606:3-65.

60. Shingfield KJ, Reynolds CK, Lupoli B, Toivonen V, Yurawecz MP, Delmonte P et $a l$. Effect of forage type and proportion of concentrate in the diet on milk fatty acid composition in cows given sunflower oil and fish oil. Anim Sci 2005;80:225-238. 